\title{
Probing the surface of aqueous surfactant-perfume mixed solutions during perfume evaporation
}

\author{
J. Penfold ${ }^{1,2}{ }^{*}$, R.K. Thomas ${ }^{1}$, R. Bradbury. ${ }^{1 \dagger}$, I. Tucker ${ }^{3}$, J.T, Petkov ${ }^{3+}$, C.W. Jones ${ }^{3}$, J.R.P. \\ Webster $^{2}$
}

1. Physical and Theoretical Chemistry Laboratory, Oxford University, South Parks Road, Oxford, OX1 3QZ, UK

2. ISIS, STFC, Rutherford Appleton Laboratory, Chilton, Didcot, Oxon, OX11 0QX, UK

3. Unilever Research and Development Laboratory, Port Sunlight, Quarry Road East, Bebington, Wirral, CH63 2JW, UK

$\dagger$ Current address: Centre for Exploitation of Energy and Matter, Dept. of Physics, Indiana University, Bloomington, In, USA

† Current address: KLK Oleo, SDN BHD, Menara KLK, Mutiara Damansara, 47810 Petaling, Jaya Selanger, Malaysia

* Corresponding Author: Jeff Penfold, Phone: +44 1235 445681, email: jeff.penfold@stfc.ac.uk

Keywords: Perfume adsorption, perfume evaporation, adsorption kinetics, air-solution interface 


\begin{abstract}
The rate of release or evaporation of perfume molecules from surfaces is a key factor in determining the impact of the perfume in a range of applications relating to home and personal care products. For mixtures of the anionic surfactant sodium dodecylsulfate and the model perfume linalool the rate of change of adsorption with time due to forced air flow over a fixed headspace was evaluated using neutron reflectivity over a period of up to $\sim 250$ mins. The measurements were made in the limit of the high flow rates where the evaporation is independent of the air flow rate. The amount of perfume at the interface decreases with time, and this occurs at a faster rate as the initial amount of perfume in solution is decreased. The variation of the perfume adsorption with time for different surfactant / perfume solution compositions illustrates the increasing importance of diffusion to the surface as the perfume concentration in solution decreases.
\end{abstract}




\section{INTRODUCTION}

Most surfactant based home and personal care products contain perfumes as a key element of their performance (1-3), and this has stimulated extensive research into the different properties of a range of model perfume molecules. The solubilisation of different perfume molecules in a range of surfactant and mixed surfactant solutions has been studied (4-8), their location within aggregates identified (9-11), and their impact upon surfactant phase behaviour determined (1215). Optimised delivery is an important element and this has stimulated the development of novel encapsulation and delivery systems (16-19).

In optimising the performance of perfumes in many of the formulations, their efficient delivery to, and retention at surfaces are important factors to understand and optimise. A number of recent studies using neutron reflectivity, NR, have focussed on these aspects. Bradbury et al (20, 21) and Penfold et al (22) have investigated the co-adsorption at the air-solution interface of some model perfume molecules, benzyl alcohol, phenylethanol, and linalool with different anionic surfactants, and notably sodium dodecylsulfate, SDS, and sodium dodecylbenzenesulfonate, LAS, and the cationic surfactant, cetyltrimethyl ammonium bromide, $\mathrm{CTAB}$. More recently potential mechanisms or routes to enhance or manipulate perfume delivery to interfaces using surface multilayer structures (23) and polyelectrolyte-surfactant surface interactions (24) have been explored.

Perfume retention and evaporation from surfaces are key aspects of perfume performance in a range of home and personal care products (1-3). The focus of this paper is on the evaporation of perfume molecules from the aqueous surface of a surfactant-perfume solution, and how it is related to the surface adsorption properties. Evaporation from surfaces has been extensively studied by indirect methods which are based on using gravimetric measurements or spectroscopic analysis of the headspace using chromatography or GC-Mass Spectroscopy (25$30)$. Beverley et al $(25,26)$ have described a gravimetric method for the determination of evaporation rates for pure liquids and liquid mixtures, in which the evaporation rates are controlled by forced gas flow over a stagnant headspace. This approach was then applied to study the selective retardation of perfume oils from oil-in-water emulsions stabilised by surfactants or nanoparticles (27).

In this paper a new approach, in which the amount of perfume adsorption at the surface during evaporation is directly measured, is presented for the first time. We demonstrate how neutron 
reflectivity from the air-solution interface of a surfactant-perfume mixed solution can be used to follow the changes in adsorption during perfume evaporation from the surface. By selective deuterium labelling of the perfume molecules the variation in neutron reflectivity with time relates directly to the time dependence of the amount of perfume at the interface. This is in turn related to the mechanism for evaporation from the surface of the perfume and its delivery to the surface from the bulk solution, which has been determined for the model perfume, linalool, LL, in the presence of the anionic surfactant SDS, at different SDS / linalool compositions. Although the methods based on gravimetric measurements and headspace analysis has provided important insights into perfume and other volatile component evaporation, the approach adopted here provides the opportunity to probe directly the role of the surface and delivery to the surface region on the evaporation process.

\section{EXPERIMENTAL DETAILS}

\section{Neutron reflectivity}

The neutron reflectivity, NR, measurements were made on the INTER reflectometer (31) at the ISIS neutron source. The reflectivity, $\mathrm{R}(\mathrm{Q})$, was measured as a function of the wave vector transfer, $\mathrm{Q}$, perpendicular to the surface (where $\mathrm{Q}=4 \pi \sin \theta / \lambda, \theta$ is the grazing angle of incidence and $\lambda$ the neutron wavelength). An accessible $Q$ range $\sim 0.03$ to $0.35 \AA^{-1}$ was covered using a $\theta$ of $2.3^{\circ}$ and a $\lambda$ range of 1 to $15 \AA$. The measurements were made at the air-water interface using a stainless steel trough to avoid excessive perfume adsorption on the trough surfaces, as des cribbed previously (20-22). A fixed headspace volume with a series of baffles was attached to the trough, and the air flow through the headspace was at a fixed rate of 40 litres / min. The trough volume was $\sim 25 \mathrm{~mL}$ and the sample temperature was $25 \pm 1^{\circ} \mathrm{C}$. The samples were aligned and the data corrected and normalised to the direct beam and the reflectivity from a deuterium oxide, $\mathrm{D}_{2} \mathrm{O}$, surface using established procedures. Each individual measurement was made for a fixed counting period, in the range 6 to 15 mins, with a continuous air flow through the headspace above the trough.

The NR measurements were made for a combination of h-SDS and d-LL in null reflecting water, nrw, (water with a neutron refractive index identical to air, 92 mole $\% \mathrm{H}_{2} \mathrm{O} / 8$ mole $\% \mathrm{D}_{2} \mathrm{O}$ ). Under these conditions the reflectivity is dominated by the presence of the deuterium labelled component at the interface, and the reflectivity is then directly related to the adsorbed amount of 
the deuterium labelled component (32), as used extensively in surfactant and mixed surfactant adsorption studies

\section{Experimental arrangement}

The stainless steel trough, with sample volume $\sim 25 \mathrm{~mL}$, is mounted in a sealed container in which the usual lid is replaced by a fixed headspace containing a series of baffles, as illustrated in figure 1.

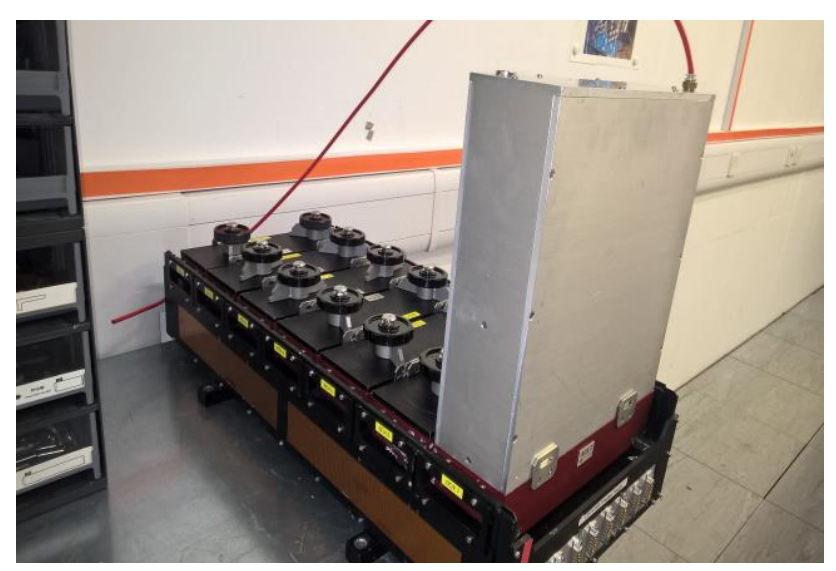

Figure 1. Experimental arrangement showing trough and headspace box.

The detailed design and layout of the headspace and associated baffles is illustrated in figure 2 . The distance between the liquid surface in the trough and the first baffle of the headspace box is $35 \mathrm{~mm}$.

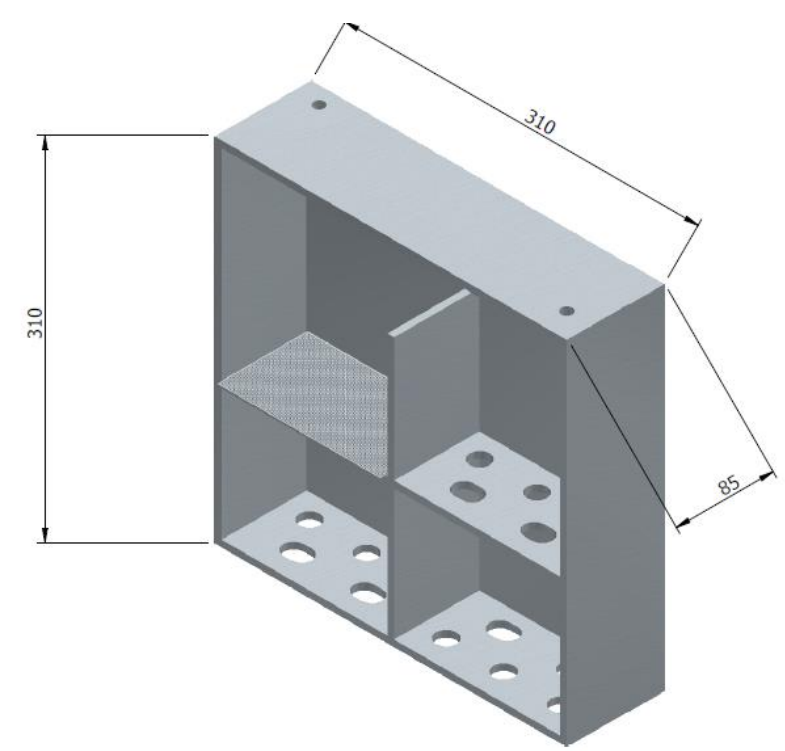

Figure 2. Schematic of headspace volume and baffles (the dimensions are in $\mathrm{mm}$ ) 
The design and layout of the baffles allows the headspace volume to be continuously replaced by a pump at a rate of 40 litres / min without any disturbance of the free liquid sample surface below it. Within the timescale of the NR measurements, up to $~ 300$ mins, the meniscus and surface of $\mathrm{D}_{2} \mathrm{O}$ or a surfactant solution are unaffected by the air flow. This is indicated, in the absence of more volatile components, by an invariance in the NR data for a $\mathrm{D}_{2} \mathrm{O}$ surface and an adsorbed layer of deuterium labelled surfactant in nrw with time. At longer pumping times than 300 mins the forced air flow at 40 lit / min was eventually sufficient to start to deplete the liquid surface level, resulting in a progressive misalignment of the incident neutron beam and an apparent reduction in the reflected signal. Hence data collection times were limited to a maximum $\leq 300$ mins to ensure that this additional complication was avoided.

\section{Materials and measurements made.}

The hydrogeneous SDS (h-SDS) was obtained from the ISIS Isotope Facility (33), and synthesised and recrystallized from ethanol as previously described (34). The purity of the SDS was verified by the absence of a minimum in the surface tension at the critical micelle concentration, cmc. The deuterium labelled linalool, $\mathrm{d}_{17}-\mathrm{LL}$ (abbreviated as $\mathrm{d}-\mathrm{LL}$ ), was synthesised at Unilever R and D (35). UHQ water (Elga Ultrapure) and $\mathrm{D}_{2} \mathrm{O}$ from Sigma was used throughout. The stainless steel trough and glassware associated with sample preparation were cleaned in Decon90 solution and rinsed thoroughly in UHQ water.

The structure of the perfume and surfactant are illustrated in figure 3, and the key parameters required in this study are summarised in table 1.<smiles>C=CC(C)(O)CCC=C(C)C</smiles>

(a) Linalool

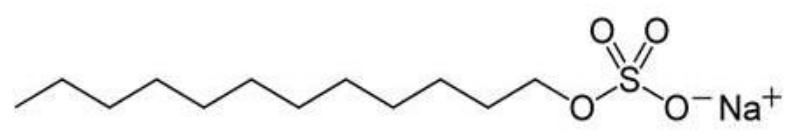

(b) Sodium dodecylsulfate

Figure 3. Molecular structure of Linalool, and sodium dodecylsulfate. 
Table 1. Key parameters for surfactant and perfume components

\begin{tabular}{|c|c|c|c|}
\hline Component & MW (g/mol) & $\sum \mathbf{b}\left(\mathbf{x 1 0}^{-3} \mathbf{\AA}\right)$ & $\begin{array}{c}\text { Vapour pressure, } \mathbf{P} \\
\text { (torr) }\end{array}$ \\
\hline h-SDS & 288 & 0.16 & - \\
\hline d-LL & 165 & 1.92 & $0.17+$ \\
\hline
\end{tabular}

+ Value taken from Sigma-Aldrich product data sheets for LL

All the NR measurements were made in nrw for the isotopic combination h-SDS / d-LL. The measurements were made at 6 to 15 min intervals; representing the minimum time step to obtain data of sufficient statistical quality to reliably determine the adsorbed amount at the interface. The measurements were made for a $1 \mathrm{mM}$ solution for SDS / linalool compositions of 60/40, 80/20, 90/10, and 96/4 mole ratio. At the solution concentration and compositions studied the solutions were below the critical micellar concentration, cmc, and so the surfactant and perfume exist only as monomers in the solution. All the measurements (unless otherwise stated) were made with a constant air flow of 40 litres / min through the headspace above the liquid surface.

\section{RESULTS and DISCUSSION}

NR measurements were made for a $1 \mathrm{mM}$ h-SDS / d-LL mixture in nrw at four different solution compositions, 60/40, 80/20, 90/10, and 96/4 mole ratio. The reflectivity data are all well described as a single monolayer with a thickness $\sim 20 \pm 2 \AA$, consistent with previous observations (21). The data were all modelled as a single layer using the exact optical matrix approach $(36,37)$ to obtain the product $d \rho$ (where $d$ is the layer thickness in $\AA$ and $\rho$ is the scattering length density in $\AA^{-3}$ of the layer). Figure 4 shows some representative NR data, for a solution mixture of $1 \mathrm{mM}$ 90/10 mole ratio h-SDS / d-LL in nrw at time zero and after 233 minutes of surface exposure to air flow. The data are described by a single monolayer, modelled with $\mathrm{d}$ and $\rho$ values of $17 \AA$ and $1.85 \times 10^{-6} \AA^{-3}$ and $16 \AA$ and $1.45 \times 10^{-6} \AA^{-3}$ at $\mathrm{t}=0$ and 233 mins respectively. When corrections are made for the h-SDS component at the interface (see discussion following) this results in adsorbed amounts of $2.48 \times 10^{-10} \mathrm{~mol} \mathrm{~cm}^{-2}$ and $1.77 \times 10^{-10}$ mol cm $\mathrm{cm}^{-2}$ respectively. 


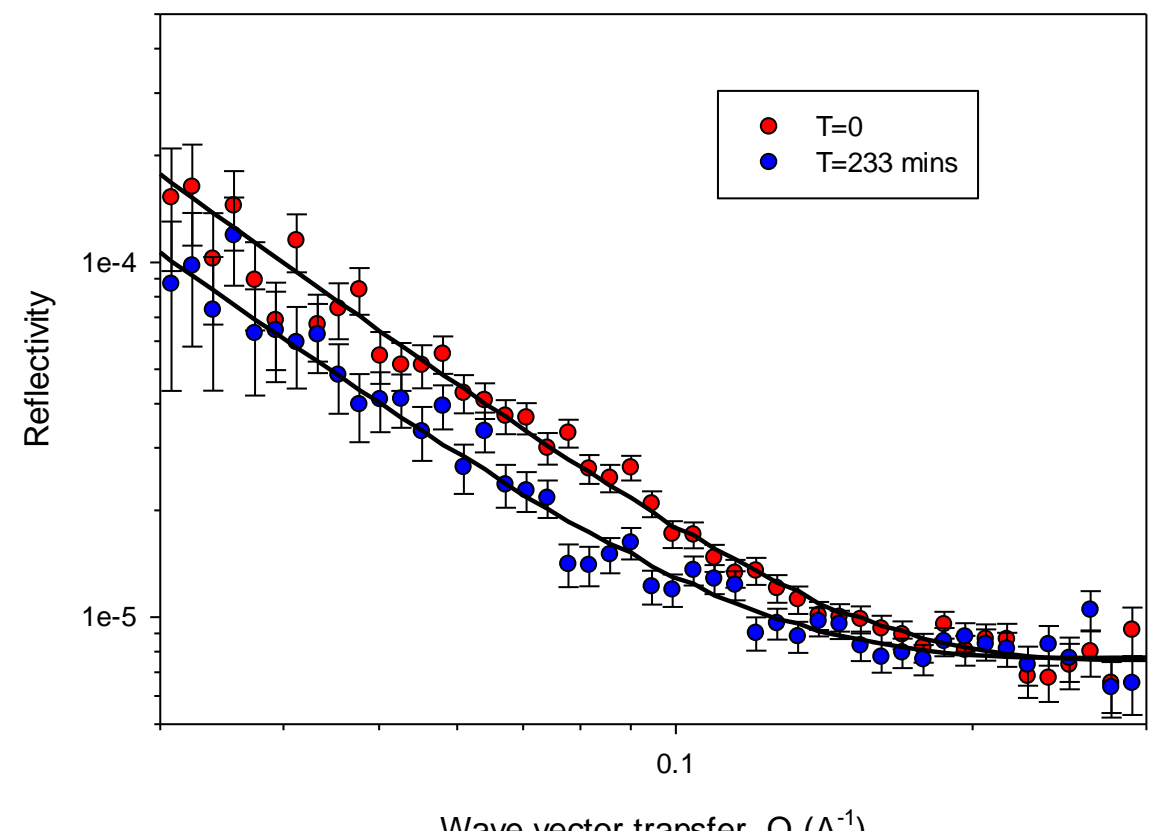

Figure 4. NR data for $1 \mathrm{mM}$ 90/10 mole ratio $h$-SDS / d-LL in nrw for (•) time=0, and (•) time+233 mins. The solid lines are model calculations for a single thin monolayer (see text for details).

For a binary mixture the adsorbed amount of each component is usually obtained by making two measurements in nrw with each component in turn deuterium labelled. The area / molecule and hence the adsorbed amount is then determined from (32),

$$
d \rho=\frac{\sum b_{1}}{A_{1}}+\frac{\sum b_{2}}{A_{2}}
$$

where $\sum b_{i}$ is the sum of scattering lengths of the two components (see table 1), and $A_{1}$ and $A_{2}$ are the area/molecule (in $\AA^{2}$ ) of the two components, surfactant and perfume, at the interface. The adsorbed amount, $\Gamma$ in mol cm$~^{-2}$, is then calculated from $\Gamma=1 / \mathrm{N}_{\mathrm{a}} \mathrm{A}$, and $\mathrm{N}_{\mathrm{a}}$ is Avogadros number. In the time dependent measurements presented here the reflectivity measurements were only made for the isotopic combination h-SDS / d-LL, in order to minimise the time interval between measurements. Although the reflectivity is dominated by the deuterium labelled LL at the interface, there is a finite contribution from the h-SDS at the interface, and this is subtracted as a fixed value from the data using equation 1. From previous studies (21) the composition of the surface layer for this mixture has been determined in full at a concentration of $1 \mathrm{mM}$ (see 
figure 2 and table S1 in the Supporting Information in reference 21). In the composition range in that study (for SDS / LL compositions of $80 / 20$ and richer in LL) the amount of SDS at the interface is relatively constant, $\Gamma \sim 2.8 \times 10^{-10} \mathrm{~mol} \mathrm{~cm}^{-2}$ (area/molecule $\sim 59 \AA^{2}$ ), and hence the effective d. $\rho$ contribution from the h-SDS is $\sim 0.27 \times 10^{-5} \AA^{-2}$. Even if the SDS composition increases as the solution decreases in perfume content the area/molecule of the SDS at the surface can only increase to a maximum value $\sim 40 \AA^{2}$, that of a pure SDS layer at saturation. This would correspond to a d $\rho$ contribution from the h-SDS $\sim 0.4 \times 10^{-6} \AA^{-2}$. For the NR data at a solution composition of $80 / 20$ mole ratio SDS / LL, for example, the values of d. $\rho$, uncompensated for the h-SDS contribution, varies from $~ 3.2$ to $2.6 \pm 0.2 \times 10^{-5} \AA^{-2}$ with time. Hence the range in the potential values for the h-SDS contribution fall within the errors quoted for the perfume adsorption.

The variations in the adsorbed amount, when corrected for the h-SDS contribution to the reflectivity, at the interface with time for $1 \mathrm{mM}$ SDS / LL and at solution compositions of 60/40, $80 / 20,90 / 10$ and 96/4 are shown in figure 5.

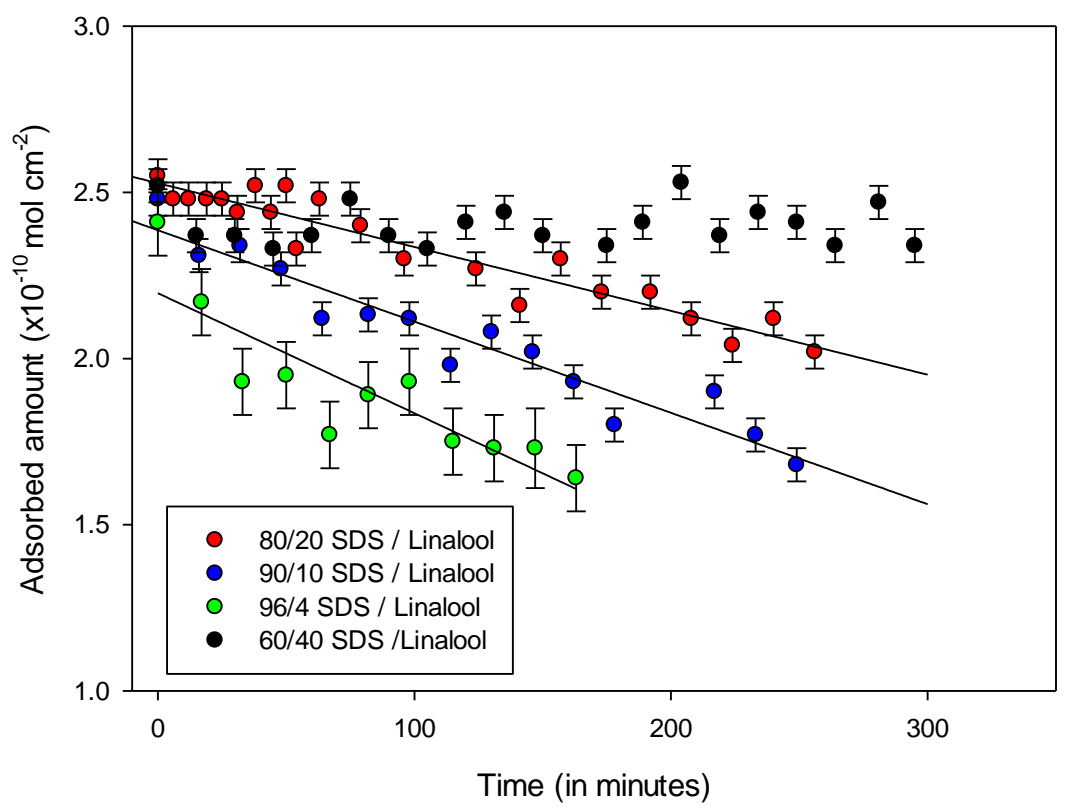

Figure 5. Variation in the LL adsorption with time for $1 \mathrm{mM} S D S$ / LL at the air-water interface, (black) 60/40, (red) 80/20, (blue) 90/10, and (green) 96/4 mole ratio SDS / LL, see also legend. The solid lines are straight line fits to the data (see text). 
During the time dependent measurements the air in the sample headspace volume is continuously exchanged at a rate of 40 litres / min. Prior to these measurements, the reflectivity was recorded in the absence of air flow; at a 80/20 mole ratio, for example, and the amount of LL at the interface was $\sim 2.6 \times 10^{-10} \mathrm{~mol} \mathrm{~cm}^{-2}$, compared to that reported by Bradbury et al (21) $\sim 2.3 \times 10^{-10} \mathrm{~mol} \mathrm{~cm}^{-2}$. Compared to the quoted error of $\pm 0.1 \times 10^{-10} \mathrm{~mol} \mathrm{~cm}^{-2}$ this is in reasonably good agreement. Similar equivalent adsorbed amounts are obtained for the solution compositions 60/40, 90/10 and 96/4 (see figure 5) and they are all consistent with the trends reported by Bradbury et al (21).

For the solution composition of $60 / 40$ mole ratio SDS / LL there is no change in the adsorbed amount of LL at the interface with increasing time during the exchange of the sample headspace by air flow over the timescale of the measurements. This implies that for the solution composition of $60 / 40$ the delivery of perfume to the surface from the bulk solution at least matches the rate at which it is removed from the surface through evaporation. Furthermore the invariance with time over the timescale of the measurements, $\sim 250$ mins, implies that the evaporation of perfume has not sufficiently depleted the solution concentration of perfume to an extent that the amount adsorbed at the interface is eventually affected. From previous equilibrium studies (21), and the equilibrium values measured here, see figure $\mathrm{S} 1$ in the Supporting Information, this would require that the solution composition was reduced to well below a solution composition of $96 / 4\left(\leq 2 \times 10^{-5} \mathrm{M}\right)$ before the LL adsorption was significantly reduced on solution depletion grounds alone.

For solution compositions of $80 / 20,90 / 10$ and $96 / 4$ the amount of perfume at the interface decreases with increasing time. The decrease is approximately linear over the timescales measured and the rate of decrease increases as the initial solution concentration of perfume is reduced. The solid lines in figure 5 are straight line fits to the data, and the corresponding slopes are $2.0 \pm 0.2,2.8 \pm 0.2$, and $3.6 \pm 0.4 \times 10^{-13} \mathrm{~mol} \mathrm{~cm}^{-2} \mathrm{~min}^{-1}$ for the solution compositions of 80/20, 90/10 and 96/4 respectively. The variation in the slope of the rate of change of adsorption with time with the initial perfume solution concentration (albeit for only 3 data points) has an exponential dependence (see figure 6), with an exponent of -3.7.

Beverley et al (25-27) used a gravimetric method to measure the evaporation from different fluid interfaces, in which the evaporation was described as limited by the evaporation and vapour diffusion across a stagnant headspace. An analytical expression was derived to describe the 
evaporation rate of a volatile fluid surface in forced air flow over a stagnant headspace. In the limit of high flow rates the evaporation is independent of flow rate, and this is the situation encountered in the experimental measurements presented here for the flow rate used.

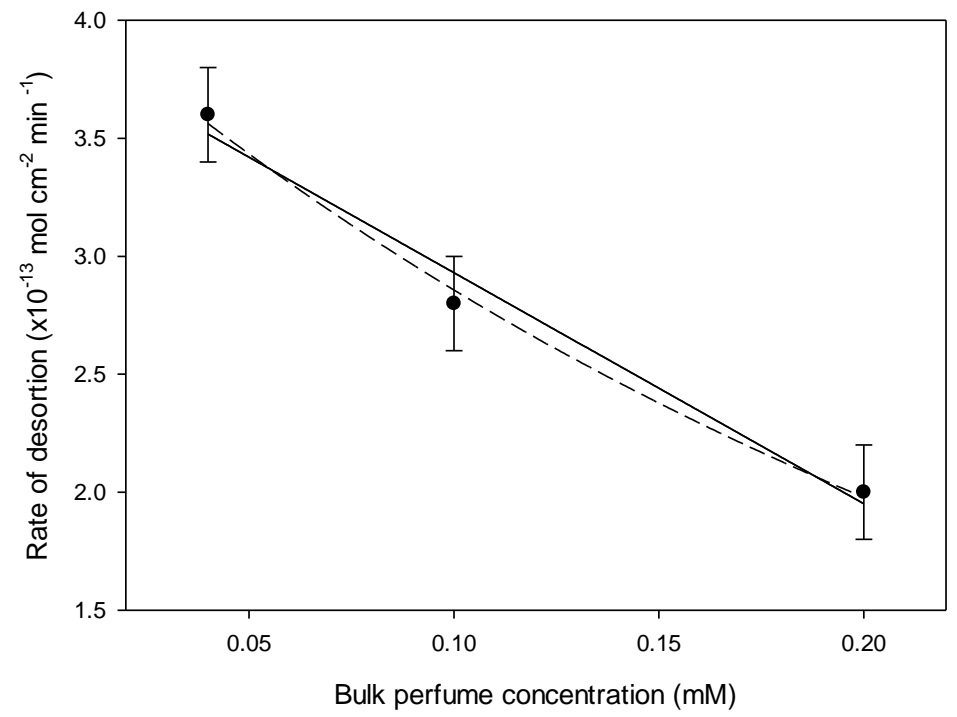

Figure 6. Variation in rate of change of adsorption with solution concentration of linalool, (-) straight line fit, (---) exponential fit.

In the NR measurements reported here an evaporation rate has been estimated from the rate of change in the adsorption with time, and using the approach of Beverley et al (25) a headspace dimension has been estimated (see Supporting Information for details of the calculation). However, as presented in the Supporting Information, the variation in the rate of change in the adsorption with solution composition implies a varying headspace dimension ( 10 to $16 \mathrm{~mm}$ ) with solution composition. Furthermore the values of the headspace dimension estimated are small compared to the physical dimension (compared to $35 \mathrm{~mm}$ ). From these observations it is assumed that in the case presented here the vapour diffusion across a stagnant headspace is not the rate limiting factor.

There are a number of other factors which may be relevant or important. The diffusion rate of the volatile component in solution to the surface may be an important factor, and we will return to this point later in the discussion. There may also be an energy barrier which affects the volatile component entering or leaving the surface, and there may well be composition or concentration gradients within the solution which affect diffusion rates. For example, Beverly et al $(26,27)$ have shown how structured liquids, colloidal gels, surfactant mesophases, and the 
presence of surfactant or nanoparticle oil-in-water emulsions can dramatically reduce evaporation rates. In ternary ethanol-water-fragrance mixtures, Tchakalova et al (30) showed how perfume evaporation kinetics depends greatly upon the solution phase behaviour and the amount and nature of the fragrance solubilisation in the different phases, such that enhancement and retardation of fragrance evaporation can occur. Behan and Parry (38) investigated the headspace concentration of perfume / surfactant (SDS) aqueous solutions using gas chromatography. They showed that the headspace concentration was directly related to the water / SDS phase volume ratio and could be described in terms of a simple partitioning. Their calculated apparent activity coefficients decreased with increasing SDS concentration. This was attributed to an increase in the perfume solubility and changes in competition for the interface. It was concluded that a more sophisticated treatment required a more detailed knowledge of the perfume-surfactant interaction. This is broadly consistent with the observations reported here, and measuring directly the variation in the adsorption during evaporation provides some additional insight into the role of the surfactant-perfume interaction.

In the measurements reported here the SDS / LL solutions are below the mixed cmc, and hence the surfactant and perfume exist only as monomers in solution. Hence it is unlikely that complicating factors due to self-assembly, solubilisation or variations in solubilisation are present in this study. It is also shown here that the equilibrium adsorption (see figure $\mathrm{S} 1$ in the Supporting Information), from 96/4 to 60/40 mole ratio SDS / LL, varies only slightly over that composition range, and indicates that the relative surface activities of the two components are not changing dramatically. Here the decrease in the adsorption with increasing time and the variation in the rate with solution composition strongly supports an interpretation that the replenishment of the surface from the solution whilst the surface is undergoing evaporation is limited by diffusion of the volatile component to the surface. That is, diffusion from solution to the surface layer or surface region depleted of perfume due to evaporation from the surface and diffusion through that depleted region will be the rate determining factors for the variation of adsorption with time. In essence there will be a concentration or composition gradient in the bulk solution near the surface, in the form of a near surface depleted region, which is affecting the diffusion rate to the surface.

Morgan et al (39) recently developed a complex numerical model to describe the kinetics of desorption from the air-solution interface resulting from the dilution of the sub-phase by laminar flow exchange. The model describes the desorption process as controlled by monomer diffusion 
from a surface boundary layer which is initially unaffected by the solvent exchange. There are broad similarities between the work of Morgan et al (38) and the experimental arrangement exploited here and the results presented. This suggests that a full numerical description of the process is feasible and now the experimental methodology has been demonstrated and established this and the exploration of a wider range of systems and conditions will the focus of future work.

The experimental arrangement and approach developed here could be applied to a wider range of systems containing volatile components, and also applied to control and measure other evaporation driven phenomena, such as the formation of surface mesophase structures by surface evaporation $(40,41)$.

\section{SUMMARY}

Perfume retention and evaporation from surfaces are key aspects of perfume performance in a range of surfactant based home and personal care products (1-3). We have presented here a novel approach using NR to measure the rate of evaporation from a free aqueous surface under forced air flow. The approach has been evaluated for the surfactant / perfume mixture of SDS / LL, for which the equilibrium adsorption properties have been previously studied and characterised (21). It has been shown that by monitoring the adsorption at the interface directly during perfume evaporation a greater insight into the factors determining the evaporation process is accessible. The results demonstrate that the rate of change in the surface adsorption of the perfume is limited by the diffusion rate of monomer through a depleted surface region to the surface. Hence the approach offers some advantages over the classical headspace analysis methods which just determine evaporation rates, and as such the underlying mechanisms may be masked.

\section{ACKNOWLEDGEMENTS}

We acknowledge the use of the reflectometer INTER at ISIS, the provision of beam time by STFC. The technical assistance on INTER of Andy Church, Jeff Vine and Tom Charleston was invaluable in setting up the headspace equipment and providing the photograph and drawing of the equipment in figure 2. 


\section{AUTHOR INFORMATION}

Corresponding author: jeff.penfold@stfc.ac.uk

Author Contributions: All the authors have given their approval of the final version of the manuscript.

Funding Sources: The neutron beam time was provided by STFC's ISIS facility.

Supporting Information: Supporting Information by way of a supplementary table, figure and discussion of the calculation of evaporation rates are available, and can be found in the online version of the paper. 


\section{REFERENCES}

(1) S Hermon, Fragrance in emulsions and surfactant systems, Cosmet. Toiletries Mag. 2006, 121, 59-67

(2) S E Friberg, Fragrance compounds and amphiphilic association structures, Adv. Coll. Int. Sci. $1998,75,181-214$

(3) P Aitkens, S E Friberg, Organised assemblies for cosmetics and transdermal drug delivery, Curr. Opin. Coll. Int. Sci. 1996, 1, 672-676

(4) Y Kondo, M Abe, K Ogino, H Uchiyama, J F Scamehorn, E E Tucker, S D Christian, Solubilisation of 2-phenylethanol in surfactant vesicles and micelles, Langmuir, 1993, 9, 899-902

(5) Y Tokuoka, H Uchiyama, M Abe, K Ogino, Solubilisation of synthetic perfumes by nonionic surfactants, J. Coll. Int. Sci. 1992, 152, 402-409

(6) M Abe, K Mizuguchi, Y Kondo, K Ogino, H Uchiyama, J F Scamehorn, E E Tucker, S D Christian, Solubilisation of perfume components in pure and mixtures of surfactants, J. Coll. Int. Sci. 1993, 160, 12-23

(7) Y Tokuoka, H Uchiyama, M Abe, Solubilisation of some synthetic perfumes by anionicnonionic mixed surfactant systems, J. Phys. Chem. 1994, 96, 6167-6171

(8) Y Tokuoka, H Uchiyama, M Abe, S D Christian, Solubilisation of some synthetic perfumes by anionic-nonionic mixed surfactants, Langmuir, 1995, 11, 725-729

(9) V Suratkar, S Mahapatra, Solubilisation site of organic perfume molecules in SDS micelles: new insights from proton NMR studies, J. Coll. Int. Sci. 2000, 225, 32-35

(10) E Fischer, W Fieber, C Navarro, H Sommer, D Beneczedi, M I Velazco, M Schonhoff, Partitioning and localisation of fragrances in surfactant mixed micelles, J. Surfact. Deterg. 2009, 12, 73-84 

benzaldehyde and limonene in lamellar liquid crystals formed with block copolymers and water, J. Coll. Int. Sc, 2006, 297, 792-798

Z Zhang, S E Friberg, P A Aikens, Change of amphiphilic structures during evaporation from emulsions in surfactant-fragrance-water systems, Int. J. Cosmet. Sci. 2000, 22. 181-199 S E Friberg. A Al-Bawab, J D Sandburg, J L Barber, Q Yui, P A Aikens, Phase behaviour of a fragrance compound system: water / phenethyl alcohol / Laureth-4 / glycerol. J. Surf. Deter. 1999, 2 159-165

M Kamada, S Shimizu, K Aramaki, Manipulation of the viscosity of wormlike gels by changing the molecular structure of added perfumes, Coll. Suf. A, 2014, 458, $110-116$

R Bradbury, J Penfold, R K Thomas, I M Tucker, J T Petkov, C Jones, Impact of model perfumes on self-assembly of the anionic surfactant sodium dodecyl benzene sulfonate, Langmuir, 2013, 29, 3233-3245 S Magdassi, Delivery systems in cosmetics, Coll. Surf. 1997, 123-123, 671-679 actives for sustained release, PCCP, 2013, 15, 17727-17741

$$
\text { P S Given, Encapsulation of flavours in emulsions and beverages, Curr. Opin. }
$$
Coll. Int. Sci. 2009, 14, 43-47

$$
\text { L B Petrovic, V J Sovilj, J M Katona, J L Milanovic, Influence of polymer- }
$$
surfactant interactions on oil-water emulsion properties and microencapsulate formation. J. Coll. Int. Sci. 2010, 342, 333-339

$$
\text { R Bradbury, J Penfold, R K Thomas, I M Tucker, J T Petkov, C Jones, }
$$
Adsorption of model perfumes at the air-solution interface by coadsorption with an anionic surfactant, Langmuir, 2013, 29, 3361-3369

$$
\text { R Bradbury, J Penfold, R K Thomas, I M Tucker, J T Petkov, C Jones, The }
$$
impact of alkyl sulfate surfactant geometry and electrolyte on the coadsorption of the 
anionic surfactant with model perfumes at the air-solution interface, J. Coll. Int. Sci. 2013, 403, 84-90

J Penfold, E Staples, I Tucker, L Soubiran, R K Thomas, A comparison of the coadsorption of benzyl alcohol and phenylethanol with CTAB at the air-water interface, J. Coll. Int. Sci. 2002, 247, 397-403

R Bradbury, J Penfold, R K Thomas, I M Tucker, J T Petkov, C Jones, Enhanced perfume surface delivery to interfaces using surface multilayer structures, J. Coll. Int. Sci. 2016, 461, 352-358

R Bradbury, J Penfold, R K Thomas, I M Tucker, J T Petkov, C Jones, Manipulating perfume delivery to interfaces using polymer-surfactant interactions, J. Coll. Int. Sci. 2016, 466, 220-226

$$
\text { K J Beverley, J H Clint, P D I Fletcher, Evaporation rates of pure liquids }
$$
measured using a gravimetric technique, PCCP, 1999, 1, 149-153

K J Beverley, J H Clint, P D I Fletcher, Evaporation rates of structured and unstructured liquid mixtures, PCCP, 2000, 2, 4173-4177

B P Binks, P D I Fletcher, B L Holt, P Beaussoubre, K Wong, Selective retardation of perfume oil evaporation from oil-in-water emulsions stabilised by either surfactants or nanoparticles, Langmuir, 2010, 26, 18024-18030

(28) C Vuilleumier, I Flament, P Sauvegrain, Headspace analysis study of evaporation rates of perfume ingredients applied to the skin. Int. J. Cosmet. Sci. 1995, 17, 61-76

(29) S E Friberg, P A Aikens, Constant vapour pressure emulsion evaporation: linalool / water stabilised by Laureth 4 sulfate, J. Coll. Int. Sci. 2009, 333, 599-604

(30) V Tchakalova, Th Zemb, D Benczedi, Evaporation triggered self-assembly in aqueous fragrance-ethanol mixtures and its impact on fragrance performance, Coll. Surf. A, 2014, 460, 414-421

INTER reflectometer at the ISIS Facility, http://www.isis.stfc.ac.uk/instruments/INTER 

structure and composition, Adv. Coll. Int. Sci. 2000, 84, 143-304 ISIS Deuteration facility, http://www.isis.stfc.ac.uk/support-laboratories/ISISisotope-facility/ISIS -deuteration-facility/

$$
\text { J R Lu, A Morroco, T J Su, R K Thomas, J Penfold, Adsorption of dodecyl }
$$
sulfate surfactants with monovalent counterions at the air-water interface studied by neutron reflectivity and surface tension, J. Coll. Int. Sci. 1993, 158, 303-316

(36) O S Heavens, Optical properties of thin solid films, Dover, New York, 1991 J Lekner, Theory of reflection, Martinus Nijhoff Publishers, The Netherlands, 1987

J M Behan, K D Perry, perfume interactions with SDS solutions, Int. J. Cosmet. Sci. 1987, 9, 261-268

C E Morgan, C J W Breward, I M Griffiths, P D Howell, J Penfold, R K Thomas, I Tucker, J T Petkov, J R P Webster, The kinetics of surfactant desorption at the airsolution interface, Langmuir, 2012, 28, 17339-17348

$$
\text { H Comas-Rojas, C Enriquez-Victorero, S J Roser, K J Edler, A Perez- }
$$

Gramatigues, Self-assembly and phase behaviour in PEI: cationic surfactant aqueous mixtures forming mesostructured films at the air/solution interface, Soft Matter, 2013, 9, $534-539$

B M D O’Discoll, E Milsom, C Fernandez-Martin, L White, S J Roser, K J Edler, Thin films of polyethyleneimine and alkyltrimethylammonium bromides at the air-water interface, Macromolecules, 2005, 38, 8785-8794 
Graphical Abstract

\section{Probing the surface of aqueous surfactant perfume mixed solutions during perfume evaporation}

J. Penfold, R.K. Thomas, R. Bradbury, I. Tucker, J.T, Petkov, C. Jones, J.R.P. Webster

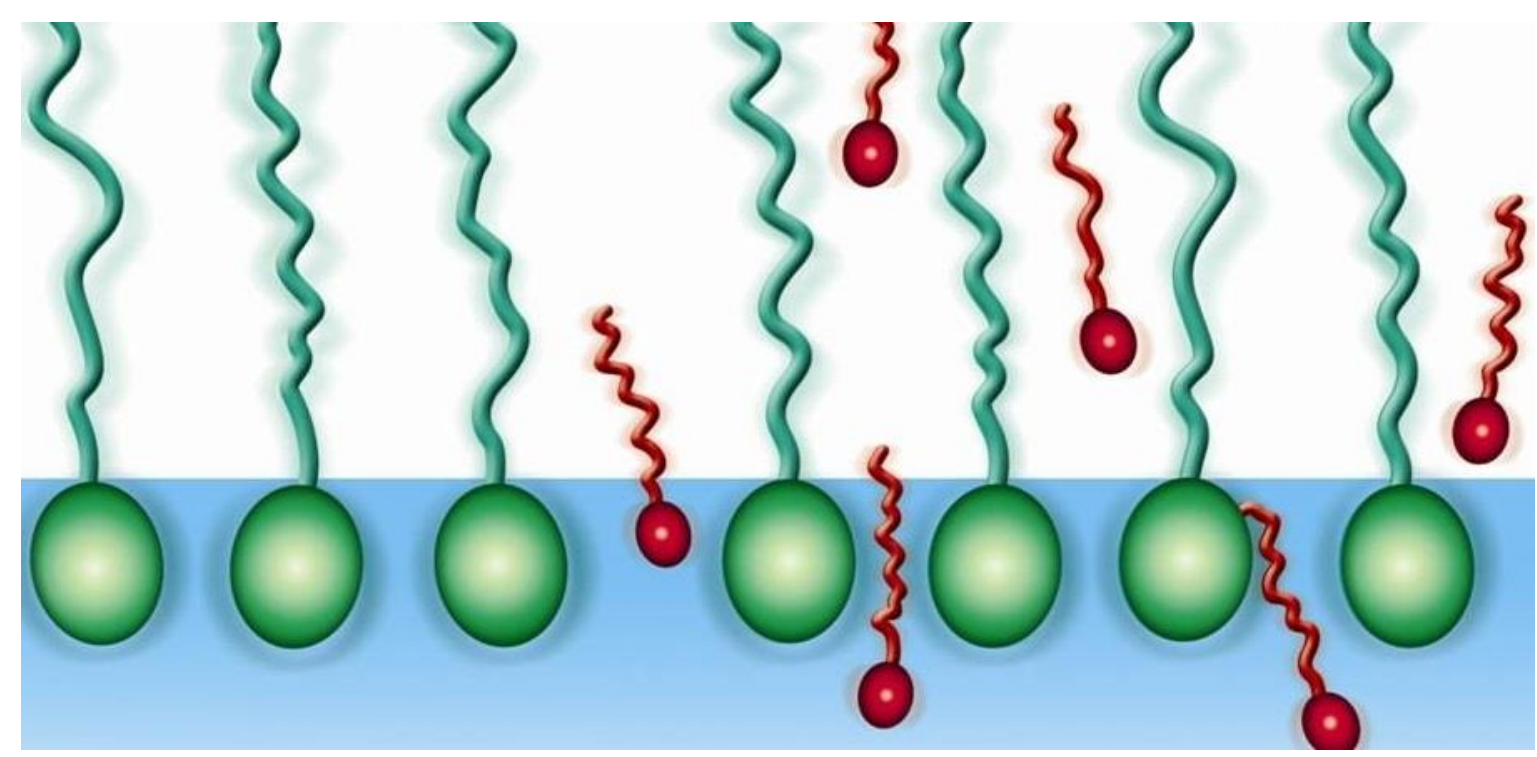

